University of Texas at El Paso

ScholarWorks@UTEP

$7-2016$

\title{
How to Explain Ubiquity of Constant Elasticity of Substitution (CES) Production and Utility Functions Without Explicitly Postulating CES
}

\author{
Olga Kosheleva \\ The University of Texas at El Paso, olgak@utep.edu \\ Vladik Kreinovich \\ The University of Texas at El Paso, vladik@utep.edu \\ Thongchai Dumrongpokaphan \\ Chiang Mai University, tcd43@hotmail.com
}

Follow this and additional works at: https://scholarworks.utep.edu/cs_techrep

Part of the Computer Sciences Commons

Comments:

Technical Report: UTEP-CS-16-45

\section{Recommended Citation}

Kosheleva, Olga; Kreinovich, Vladik; and Dumrongpokaphan, Thongchai, "How to Explain Ubiquity of Constant Elasticity of Substitution (CES) Production and Utility Functions Without Explicitly Postulating CES" (2016). Departmental Technical Reports (CS). 1016.

https://scholarworks.utep.edu/cs_techrep/1016

This Article is brought to you for free and open access by the Computer Science at ScholarWorks@UTEP. It has been accepted for inclusion in Departmental Technical Reports (CS) by an authorized administrator of ScholarWorks@UTEP. For more information, please contact Iweber@utep.edu. 


\title{
How to Explain Ubiquity of Constant Elasticity of Substitution (CES) Production and Utility Functions Without Explicitly Postulating CES
}

Olga Kosheleva, Vladik Kreinovich, and Thongchai Dumrongpokaphan

\begin{abstract}
In many situations, the dependence of the production or utility on the corresponding factors is described by the CES (Constant Elasticity of Substitution) functions. These functions are usually explained by postulating two requirements: an economically reasonable postulate of homogeneity (that the formulas should not change if we change a measuring unit) and a less convincing CSE requirement. In this paper, we show that the CES requirement can be replaced by a more convincing requirement - that the combined effect of all the factors should not depend on the order in which we combine these factors.
\end{abstract}

\section{Formulation of the Problem}

CES production functions and CES utility function are ubiquitous. Most observed data about production $y$ is well described by the CES production function

$$
y=\left(\sum_{i=1}^{n} a_{i} \cdot x_{i}^{r}\right)^{1 / r}
$$

where $x_{i}$ are the numerical measures of the factors that influence production, such as amount of capital, amount of labor, etc.; see, e.g., [5, 16, 17, 23].

Olag Kosheleva

University of Texas at El Paso, 500 W. University, El Paso, Texas 79968, USA,

e-mail: olgak@utep.edu

Vladik Kreinovich

Department of Computer Science, University of Texas at El Paso, 500 W. University, El Paso, Texas 79968, USA, e-mail: vladik@utep.edu

Thongchai Dumrongpokaphan

Department of Mathematics, Faculty of Science, Chiang Mai University, Thailand,

e-mail: tcd43@hotmail.com 
A similar formula (1) describes how the person's utility $y$ depends on different factors $x_{i}$ such as amounts of different types of consumer goods, utilities of other people, etc.; see, e.g., [6, 11, 12, 28].

How this ubiquity is explained now. The current explanation for the empirical success of CES function is based on the following two requirements.

The first requirement is that the corresponding function $y=f\left(x_{1}, \ldots, x_{n}\right)$ is homogeneous, i.e., that:

$$
f\left(\lambda \cdot x_{1}, \ldots, \lambda \cdot x_{n}\right)=\lambda \cdot f\left(x_{1}, \ldots, x_{n}\right) .
$$

This requirement makes perfect economic sense: e.g., we can describe different factors by using different monetary units, and the results should not change if we replace the original unit by a one which is $\lambda$ times smaller. After this replacement, the numerical value of each factor changes from $x_{i}$ to $\lambda \cdot x_{i}$ and $y$ is replace by $\lambda \cdot y$. The value $f\left(\lambda \cdot x_{1}, \ldots, \lambda \cdot x_{n}\right)$ that we obtain by using the new units should thus be exactly $\lambda$ times larger than the value $f\left(x_{1}, \ldots, x_{n}\right)$ obtained in the original units and this is exactly the requirement (2).

The second requirement is that the corresponding function $f\left(x_{1}, \ldots, x_{n}\right)$ should provide constant elasticity of substitution (CES). The requirement is easier to explain for the case of two factors $n=2$. In this case, this requirement deals with "substitution" situations in which we change $x_{1}$ and then change the original value $x_{2}$ to the new value $x_{2}\left(x_{1}\right)$ so that the overall production or utility remain the same.

The corresponding substitution rate can then be calculated as $s \stackrel{\text { def }}{=} \frac{d x_{2}}{d x_{1}}$. The substitution function $x_{2}\left(x_{1}\right)$ is explicitly defined by the equation $f\left(x_{1}, x_{2}\left(x_{1}\right)\right)=$ const. By using the formula for the derivative of the implicit function, we can conclude that the substitution rate has the form

$$
s=-\frac{f_{, 1}\left(x_{1}, x_{2}\right)}{f_{, 2}\left(x_{1}, x_{2}\right)}
$$

where we denoted

$$
f_{, 1}\left(x_{1}, x_{2}\right) \stackrel{\text { def }}{=} \frac{\partial f}{\partial x_{1}}\left(x_{1}, x_{2}\right) \text { and } f_{, 2}\left(x_{1}, x_{2}\right) \stackrel{\text { def }}{=} \frac{\partial f}{\partial x_{2}}\left(x_{1}, x_{2}\right)
$$

The requirement is that for each percent of the change in ratio $\frac{x_{2}}{x_{1}}$, we get the same constant number of percents change in $s$ :

$$
\frac{d s}{d\left(\frac{x_{2}}{x_{1}}\right)}=\text { const. }
$$


This explanation needs strengthening. While homogeneity is a reasonable requirement, the above CES condition sounds somewhat too mathematical to be fully convincing for economists.

To explain the ubiquity of CSE production and utility functions, it is therefore desirable to come up with additional - hopefully, more convincing - arguments in favor of these functions. This is what we intend to do in this paper.

\section{Main Idea Behind a New Explanation}

Main idea. In our explanation, we will use the fact that in most practical situations, we combine several factors. We can combine these factors in different order:

- For example, we can first combine the effects of capital and labor into a single characteristic that describes the joint even of both factors, and then combine it with other factors.

- Alternatively, we can first combine capital with other factors, and only then combine the resulting combined factor with labor, etc.

The result should not depend on the order in which we perform these combinations.

What we do in this paper. In this paper, we show that this idea implies the CES functions. Thus, we indeed get a new explanation for the ubiquity of CES production and utility functions.

\section{Derivation of the CES Functions from the Above Idea}

Towards formalizing our idea. Let us denote a function that combines factors $i$ and $j$ into a single quantity $x_{i j}$ by $f_{i, j}\left(x_{i}, x_{j}\right)$. Similarly, let us denote a function that combines the values $x_{i j}$ and $x_{k \ell}$ into a single quantity $x_{i j k \ell}$ by $f_{i j, k \ell}\left(x_{i j}, x_{k \ell}\right)$. In these terms, the requirement that the resulting values do not depend on the order implies, e.g., that we always have

$$
f_{12,34}\left(f_{1,2}\left(x_{1}, x_{2}\right), f_{3,4}\left(x_{3}, x_{4}\right)\right)=f_{13,24}\left(f_{1,3}\left(x_{1}, x_{3}\right), f_{2,4}\left(x_{2}, x_{4}\right)\right) .
$$

Additional requirement. In both production and utility situations, for each $i$ and $j$, the combination function $f_{i, j}\left(x_{i}, x_{j}\right)$ is an increasing function of both variables $x_{i}$ and $x_{j}$. It is reasonable to require that it is continuous, and then when one of the factors tends to infinity, the result also tends to infinity. Under these reasonable assumptions, the combination functions tends out to be invertible in the following sense: 
Definition 1. A function $f: A \times B \rightarrow C$ is called invertible if the following two conditions are satisfied:

- for every $a \in A$ and for every $c \in C$, there exists a unique value $b \in B$ for which $c=f(a, b)$;

- for every $b \in B$ and for every $c \in C$, there exists a unique value a $\in A$ for which $f(a, b)=c$.

Comment. In mathematics, functions invertible in the sense of Definition 1 are called generalized quasigroups; see, e.g., [4].

Let us now formalize the above requirement.

Definition 2. Let $X_{i}, X_{i j}$, and $X$ be sets, where $i=1,2,3,4$. We say that invertible operations $f_{i, j}: X_{i} \times X_{j} \rightarrow X_{i j}$ and $f_{i j, k \ell}: X_{i j} \times X_{k \ell} \rightarrow X$ (for different $i, j, k$, and $\ell$ ) satisfy the generalized associativity requirement if for all $x_{i} \in X_{i}$, we have

$$
f_{12,34}\left(f_{1,2}\left(x_{1}, x_{2}\right), f_{3,4}\left(x_{3}, x_{4}\right)\right)=f_{13,24}\left(f_{1,3}\left(x_{1}, x_{3}\right), f_{2,4}\left(x_{2}, x_{4}\right)\right) .
$$

Comment. In mathematical terms, this requirement is known as generalized mediality [4].

Groups and Abelian groups: reminder. To describe operations that satisfy the generalized associativity requirement, we need to recall that a set $G$ with an associative operation $g(a, b)$ and a unit element $e$ (for which $g(a, e)=g(e, a)=a$ ) is called a group if every element is invertible, i.e., if for every $a$, there exists an $a^{\prime}$ for which $g\left(a, a^{\prime}\right)=e$. A group in which the operation $g(a, b)$ is commutative is known as Abelian.

Proposition. [2, 3, 4, 25, 26, 27] For every set of invertible operations that satisfy the generalized associativity requirement, there exists an Abelian group $G$ and 1-1 mappings $r_{i}: X_{i} \rightarrow G, r_{i j}: X_{i j} \rightarrow G$ and $r_{X}: X \rightarrow G$ for which, for all $x_{i} \in X_{i}$ and $x_{i j} \in X_{i j}$, we have

$$
\begin{gathered}
f_{i j}\left(x_{i}, x_{j}\right)=r_{i j}^{-1}\left(g\left(r_{i}\left(x_{i}\right), r_{j}\left(x_{j}\right)\right)\right) \text { and } \\
f_{i j, k l}\left(x_{i j}, x_{k \ell}\right)=r_{X}^{-1}\left(g\left(r_{i j}\left(x_{i j}\right), r_{k \ell}\left(x_{k \ell}\right)\right)\right) .
\end{gathered}
$$

Discussion. All continuous 1-D Abelian groups with order-preserving operations are isomorphic to the additive group of real numbers, with $g(a, b)=a+b$. Thus, we can conclude that all combining operations have the form

$$
f_{i j}\left(x_{i}, x_{j}\right)=r_{i j}^{-1}\left(r_{i}\left(x_{i}\right)+r_{j}\left(x_{j}\right)\right)
$$

i.e., equivalently, $f_{i j}\left(x_{i}, x_{j}\right)=y$ means that

$$
r_{i j}(y)=r_{i}\left(x_{i}\right)+r_{j}\left(x_{j}\right)
$$


Let us use homogeneity: result. We will now prove that homogeneity leads exactly to the desired CES combinations. This will give us the desired new explanation of the ubiquity of the CES operations.

Homogeneity leads to CES operations: proof. Homogeneity means that if the relation (5) holds for some values $x_{i}, x_{j}$, and $y$, then, for every $\lambda$, a similar relation holds for re-scaled values $\lambda \cdot x_{i}, \lambda \cdot x_{j}$, and $\lambda \cdot y$, i.e.:

$$
r_{i j}(\lambda \cdot y)=r_{i}\left(\lambda \cdot x_{i}\right)+r_{j}\left(\lambda \cdot x_{j}\right)
$$

To utilize this requirement, let us use the idea of substitution: for each possible value $x_{i}^{\prime}=x_{i}+\Delta x_{i}$, let us find the corresponding value $x_{j}^{\prime}=x_{j}+\Delta x_{j}$ for which the righthand side of the formula (5) remains the same - and thus, the combined value $y$ remains the same:

$$
r_{i}\left(x_{1}^{\prime}\right)+r_{j}\left(x_{j}^{\prime}\right)=r_{i}\left(x_{i}+\Delta x_{i}\right)+r_{j}\left(x_{j}+\Delta x_{j}\right)=r_{i}\left(x_{i}\right)+r_{j}\left(x_{j}\right) .
$$

In general, the substitute value $x_{j}^{\prime}$ is a function of $x_{i}^{\prime}: x_{j}^{\prime}=x_{j}^{\prime}\left(x_{i}^{\prime}\right)$. When $\Delta x_{i}=$ 0 , i.e., when $x_{i}^{\prime}=x_{i}$, we clearly have $x_{j}^{\prime}=x_{j}$, so $\Delta x_{j}=0$. For small $\Delta x_{i}$, we get $y_{j}^{\prime}=y_{j}+k \cdot \Delta x_{i}+o\left(\Delta x_{i}\right)$, where $k \stackrel{\text { def }}{=} \frac{d x_{j}^{\prime}}{d x_{i}^{\prime}}$, so $\Delta x_{j}=k \cdot \Delta x_{i}+o\left(\Delta x_{i}\right)$ for some real number $k$.

Here, $r_{i}\left(x_{i}+\Delta x_{i}\right)=r_{i}\left(x_{i}\right)+r_{i}^{\prime}\left(x_{i}\right) \cdot \Delta x_{i}+o\left(\Delta x_{i}\right)$, where, as usual, $f^{\prime}$ denotes the derivative. Similarly,

$$
r_{j}\left(x_{j}+\Delta x_{j}\right)=r_{j}\left(x_{j}+k \cdot \Delta x_{i}+o\left(\Delta x_{i}\right)\right)=r_{j}\left(x_{j}\right)+k \cdot r_{j}^{\prime}\left(x_{j}\right) \cdot \Delta x_{i}+o\left(\Delta x_{i}\right) .
$$

Thus, the condition (6) takes the form

$$
r_{i}\left(x_{i}\right)+r_{j}\left(x_{j}\right)+\left(r_{i}^{\prime}\left(x_{i}\right)+k \cdot r_{j}^{\prime}\left(x_{j}\right)\right) \cdot \Delta x_{i}+o\left(\Delta x_{i}\right)=r_{i}\left(x_{i}\right)+r_{j}\left(x_{j}\right) .
$$

Subtracting the right-hand side from the both sides, dividing both sides of the resulting equation by $\Delta x_{i}$, and tending $\Delta x_{i}$ to 0 , we conclude that

$$
r_{i}^{\prime}\left(x_{i}\right)+k \cdot r_{j}^{\prime}\left(x_{j}\right)=0,
$$

i.e., that

$$
k=-\frac{r_{i}^{\prime}\left(x_{i}\right)}{r_{j}^{\prime}\left(x_{j}\right)}
$$

Homogeneity means, in particular, that if now apply the combination function $r_{i j}$ to the values

$$
\lambda \cdot x_{i}^{\prime}=\lambda \cdot x_{i}+\lambda \cdot \Delta x_{i}
$$

and

$$
\lambda \cdot x_{j}^{\prime}=\lambda \cdot x_{j}+\lambda \cdot k \cdot \Delta x_{i}+o\left(\Delta x_{i}\right)
$$


then we should get the value $\lambda \cdot y$. So:

$$
r_{i}\left(\lambda \cdot x_{i}+\lambda \cdot \Delta x_{i}\right)+r_{j}\left(\lambda \cdot x_{j}+\lambda \cdot k \cdot \Delta x_{i}+o\left(\Delta x_{i}\right)\right)=r_{i}\left(\lambda \cdot x_{i}\right)+r_{j}\left(\lambda \cdot x_{j}\right) .
$$

For small $\Delta x_{i}$, we have

$$
r_{i}\left(\lambda \cdot x_{i}+\lambda \cdot \Delta x_{i}\right)=r\left(\lambda \cdot x_{i}\right)+\lambda \cdot \Delta x_{i} \cdot r_{i}^{\prime}\left(\lambda \cdot x_{i}\right)+o\left(\Delta x_{i}\right),
$$

where $f^{\prime}$ denote a derivative, and similarly,

$$
r_{j}\left(\lambda \cdot x_{j}+\lambda \cdot k \cdot \Delta x_{i}+o\left(\Delta x_{1}\right)\right)=r\left(\lambda \cdot x_{2}\right)+\lambda \cdot k \cdot \Delta x_{i} \cdot r_{j}^{\prime}\left(\lambda \cdot x_{j}\right)+o\left(\Delta x_{i}\right) .
$$

Substituting these expressions into the formula (8), we conclude that

$$
\begin{gathered}
r_{i}\left(\lambda \cdot x_{i}\right)+\lambda \cdot \Delta x_{i} \cdot r^{\prime}\left(\lambda \cdot x_{i}\right)+r_{j}\left(\lambda \cdot x_{j}\right)+\lambda \cdot k \cdot r_{j}^{\prime}\left(\lambda \cdot x_{j}\right) \cdot \Delta x_{i}+o\left(\Delta x_{i}\right)= \\
r_{i}\left(\lambda \cdot x_{i}\right)+r_{j}\left(\lambda \cdot x_{j}\right) .
\end{gathered}
$$

Subtracting the right-hand side from the left-hand side, dividing the result by $\Delta x_{i}$ and tending $\Delta x_{i}$ to 0 , we conclude that

$$
r^{\prime}\left(\lambda \cdot x_{i}\right)+k \cdot r_{j}^{\prime}\left(\lambda \cdot x_{j}\right)=0,
$$

i.e., in view of the formula (7), that

$$
r^{\prime}\left(\lambda \cdot x_{i}\right)-\frac{r_{i}^{\prime}\left(x_{i}\right)}{r_{j}^{\prime}\left(x_{j}\right)} \cdot r_{j}^{\prime}\left(\lambda \cdot x_{j}\right)=0 .
$$

Moving the second term to the right-hand side and dividing both sides by $r_{i}^{\prime}\left(x_{i}\right)$, we conclude that

$$
\frac{r_{i}^{\prime}\left(\lambda \cdot x_{i}\right)}{r_{i}^{\prime}\left(x_{i}\right)}=\frac{r_{j}^{\prime}\left(\lambda \cdot x_{j}\right)}{r_{j}^{\prime}\left(x_{j}\right)}
$$

The right-hand side of this formula does not depend on $x_{i}$ at all, thus, the left-hand side also does not depend on $x_{i}$, it only depends on $\lambda$ :

$$
\frac{r_{i}^{\prime}\left(\lambda \cdot x_{i}\right)}{r_{i}^{\prime}\left(x_{i}\right)}=c(\lambda)
$$

for some function $c(\lambda)$. Thus, the derivative $R_{i}\left(x_{i}\right) \stackrel{\text { def }}{=} r_{i}^{\prime}\left(x_{i}\right)$ satisfies the functional equation

$$
R_{i}\left(\lambda \cdot x_{i}\right)=R_{i}\left(x_{i}\right) \cdot c(\lambda)
$$

for all $\lambda$ and $x_{i}$.

It is know that every continuous solution to this equation has the form $r_{i}^{\prime}\left(x_{i}\right)=$ $R_{i}\left(x_{i}\right)=A_{i} \cdot x_{i}^{\alpha_{i}}$ for some $A_{i}$ and $\alpha_{i}$; see, e.g., [4]. For differentiable functions, this can be easily proven if we differentiate both sides of this equation by $c$ and take $c=1$. Then, we get $x_{i} \cdot \frac{d R_{i}}{d c_{i}}=c \cdot R_{i}$. Separating variables, we get 


$$
\frac{d R_{i}}{R_{i}}=c \cdot \frac{d x_{i}}{x_{i}} .
$$

Integration leads to $\ln \left(R_{i}\right)=c \cdot \ln \left(x_{i}\right)+C_{1}$ and thus, to the desired formula.

Integrating the above expression for $r_{i}^{\prime}\left(x_{i}\right)$, we get $r_{i}\left(x_{i}\right)=a_{i} \cdot x_{i}^{\beta_{i}}+C_{i}$ and similarly, $r_{j}\left(x_{j}\right)=a_{j} \cdot x_{j}^{\beta_{j}}+C_{j}$. One can easily check that homogeneity implies that $\beta_{i}=\beta_{j}$ and $C_{i}+C_{j}=0$, so the sum $r_{i}\left(x_{i}\right)+r_{j}\left(x_{j}\right)$ takes the form $a_{i} \cdot x_{i}^{r}+a_{j} \cdot x_{j}^{r}$.

By considering a similar substitution between $x_{i}$ and $y$ (in which $x_{j}$ remains intact), we conclude that $r_{i j}(y)=$ const $\cdot y^{r}$, so we indeed get the desired formula $r_{i j}\left(x_{i}, x_{j}\right)=\left(a_{i} \cdot x_{i}^{r}+a_{j} \cdot x_{j}^{r}\right)^{1 / r}$. By using similar formulas to combine $x_{i j}$ with $x_{k}$, etc., we get the desired CES combination function.

\section{Possible Application to Copulas}

What is a copula: a brief reminder. Specifically, a 1-D probability distribution of a random variable $X$ can be described by its cumulative distribution function (cdf) $F_{X}(x) \stackrel{\text { def }}{=} \operatorname{Prob}(X \leq x)$. A 2-D distribution of a random vector $(X, Y)$ can be similarly described by its 2-D $\operatorname{cdf} F_{X Y}(x, y)=\operatorname{Prob}(X \leq x \& Y \leq y)$.

It turns out that we can always describe $F(x, y)$ as

$$
F_{X Y}(x, y)=C_{X Y}\left(F_{X}(x), F_{Y}(y)\right)
$$

for an appropriate function $C_{X Y}:[0,1 \times[0,1] \rightarrow[0,1]$ known as a copula; see, e.g., $[20,22]$.

For a joint distribution of several random variables $X, Y, \ldots, Z$, we can similarly write

$$
\begin{gathered}
F_{X Y \ldots Z}(x, y, \ldots, z) \stackrel{\text { def }}{=} \operatorname{Prob}(X \leq x \& Y \leq y \& \ldots \& Z \leq z)= \\
C_{X Y \ldots Z}\left(F_{X}(x), F_{Y}(y), \ldots, F_{Z}(z)\right)
\end{gathered}
$$

for an appropriate multi-D copula $C_{X Y} \ldots Z$.

Vine copulas. When we have many $(n \gg 1)$ random variables, then to exactly describe their joint distribution, we need to describe a general function of $n$ variables. Even if we use two values for each variable, we get $2^{n}$ combinations, which for large $n$ can be astronomically large. Thus, a reasonable idea is to approximate the multi-D distribution.

A reasonable way to approximate is to use 2-D copulas. For example, to describe a joint distribution of three variables $X, Y$, and $Z$, we first describe the joint distribution of $X$ and $Y$ as $F_{X Y}(x, y)=C_{X Y}\left(F_{X}(x), F_{Y}(y)\right)$, and then use an appropriate copula $C_{X Y, Z}$ to combine it with $F_{Z}(z)$ :

$$
F_{X Y Z}(x, y, z) \approx C_{X Y, Z}\left(F_{X Y}(x, y), F_{Z}(z)\right)=C_{X Y, Z}\left(C_{X Y}\left(F_{X}(x), F_{Y}(y), F_{Z}(z)\right) .\right.
$$


Such an approximation, when copulas are applied to one another like a vine, are known as vine copulas; see, e.g., [1, 7, 8, 10, 13, 14, 15, 18, 19, 21, 24].

Natural analogue of associativity. It is reasonable to require that the result of the vine copula approximation should not depend on the order in which we combine the variables. In particular, for four random variables $X, Y, Z$, and $T$, we should get the same result in the following two situations:

- if we first combine $X$ with $Y, Z$ and $T$, and then combine the two results; or

- if we first combine $X$ with $Z, Y$ with $T$, and then combine the two results.

Thus, we require that for all possible real numbers $x, y, z$, and $t$, we get

$$
\begin{gathered}
C_{X Y, Z T}\left(C_{X Y}\left(F_{X}(x), F_{Y}(y)\right), C_{Z T}\left(F_{Z}(z), F_{T}(t)\right)\right)= \\
C_{X Z, Y T}\left(C_{X Z}\left(F_{X}(x), F_{Z}(z)\right), C_{Y T}\left(F_{Y}(y), F_{T}(t)\right)\right) .
\end{gathered}
$$

If we denote $a=F_{X}(x), b=F_{Y}(y), c=F_{Z}(z)$, and $d=F_{T}(t)$, we conclude that for every $a, b, c$, and $d$, we have

$$
C_{X Y, Z T}\left(C_{X Y}(a, b), C_{Z T}(c, d)\right)=C_{X Z, Y T}\left(C_{X Z}(a, c), C_{Y T}(b, d)\right) .
$$

This is exactly the generalized associativity requirement. Thus, if we extend copulas to invertible operations, then we can conclude that copulas can be re-scaled to associative operations - in the sense of the above Proposition.

\section{Acknowledgments}

This work was supported in part by the National Science Foundation grants HRD0734825 and HRD-1242122 (Cyber-ShARE Center of Excellence) and DUE0926721, and by an award "UTEP and Prudential Actuarial Science Academy and Pipeline Initiative” from Prudential Foundation.

\section{References}

1. K. Aas, C. Czado, A. Frigessi, and H. Bakken, "Pair-copula constructions of multiple dependence", Insurance: Mathematics and Economics, 2009, Vol. 44, pp. 182-198.

2. J. Aczél, V. D. Belousov, and M. Hosszú, "Generalized associativity and bisymmetry on quasigroups", Acta Math. Acad. Sci, Hungar., 1960, Vol. 11, pp. 127-136.

3. J. Aczeél, "Quasigroup-net-nomograms", Advances in Mathematics, 1965, Vol. 1, pp. 483450 .

4. J. Aczél and J. Dhombres, Functional Equations in Several Variables, Camridge University Press, Cambridge, UK, 2008.

5. K. J. Arrow, H. B. Chenery, B. S. Minhas, and R. M. Solow, "Capital-labor substitution and economic efficiency", Review of Economics and Statistics, 1961, Vol. 43, No. 3, pp. 225-250. 
6. G. Baltas, "Utility-consistent brand demand systems with endogeneous category consumption: principles and marketing applications", Decision Sciences, 2001, Vol. 32, No. 3, pp. 399-421.

7. T. Bedford and R. M. Cooke, "Monte Carlo simulation of vine dependent random variables for applications in uncertainty analysis", Proceedings of European Safety and Reliability Conference ESREL'2001, Turin, Italy, 2001.

8. T. Bedford and R. M. Cooke, "Vines - a new graphical model for dependent random variables", Annals of Statistics, 2002, Vol. 30, No. 4, pp. 1031-1068.

9. B. G. Buchanan and E. H. Shortliffe, Rule Based Expert Systems: The MYCIN Experiments of the Stanford Heuristic Programming Project, Addison-Wesley, Reading, Massachusetts, 1984.

10. C. Czado, "Pair-copula constructions of multivariate copulas", In: P. Jaworski (Ed.), Copula Theory and Its Applications, Lecture Notes in Statistics, Vol. 198, Springer-Verlag, Berlin, Heidelberg, 2010, pp. 93-109.

11. A. Dixit and J. Steiglitz, "Monopolistic competition and optimum product diversity", American Economic Review, 1977, Vol. 67, No. 3, pp. 297-308.

12. R. Fisman, P. Jakiela, S. Kariv, and D. Markovits, "The distributional preferences of an elite", Science, 2015, Vol. 349, No. 6254, p. 1300 and artile aab096.

13. H. Joe, "Dependence comparisons of vine copulae with four or more variables", In: D. Kurowicka and H. Joe (Eds.), Dependence Modeling: Vine Copula Handbook, World Scientific, Singapore, 2010.

14. H. Joe and T. Hu, "Multivariate distributions from mixtures of max-infinitely divisible distributions", Journal of Multivariate Analysis, 1996, Vol. 57, No. 2, pp. 240-265.

15. H. Joe, H. Li, and A.K. Nikoloulopoulos, "Tail dependence functions and vine copulas", Journal of Multivariate Analysis, 2010, Vol. 101, pp. 252-270.

16. D. W. Jorgensen, Econometrics, Vol. 1, Economic Modelling of Producer behavior, MIT Press, Campridge, Massachusetts, 2000.

17. R. Klump, P. McAadam, and A. Willaims "Factor substitution and factor augmenting technical progress in the US: a normalized supply-side system approach", Review of Economics and Statisticsm 2007, Vol. 89, No. 1, pp. 183-192.

18. D. Kurowicka and R.M. Cooke, Uncertainty Analysis with High Dimensional Dependence Modelling, Wiley, New York, 2006.

19. D. Kurowicka and H. Joe (Eds.), Dependence Modeling: Vine Copula Handbook, World Scientific, Singapore, 2010.

20. R. B. Nelsen, An Introduction to Copulas, Springer, New York, 1999.

21. A. K. Nikoloulopoulos, "Vine copulas with asymmetric tail dependence and applications to financial return data", Computational Statistics and Data Analysis, 2012, Vol. 56, pp. 36593673.

22. A. Sklar, "Fonctions de répartition á $n$ dimensions et leurs marges", Publ. Inst. Statist. Univ. Paris, 1959, Vol. 8, pp. 229-231.

23. R. M. Solow, "A contribution to the theory of economic growth", The Quartely Journal of Economics, 1956, Vol. 70, pp. 65-94.

24. S. Sriboonchitta, O. Kosheleva, and H. T. Nguyen, "Why Are Vine Copulas So Successful in Econometrics?", International Journal of Uncertainty, Fuzziness, and Knowledge-Based Systems (IJUFKS), 2015, Vol. 23, Suppl. 1, pp. 133-142.

25. M. A. Taylor, "The generalized equations of bisymmetry, associativity and transitivity on quasigroups”, Canadian Math. Bulletin, 1972, Vol. 15, pp. 119-124.

26. M. A. Taylor, "Certian functional equations on groupoids weaker than quasigroups", Aequationes Mathematicae, 1973, Vol. 9, pp. 23-29.

27. M. A. Taylor, "On the generalized equations of associativity and bisymmetry", Aequationes Mathematicae, 1978, Vol. 17, pp. 154-163.

28. H. Varian, Microeconometric Analysis, Norton, New York, 1992. 